\title{
Aural polyp with facial asymmetry in an unfortunate infant
}

\author{
Farah Syahida Zubir, Jeyasakthy Saniasiaya, Haslinda Abdul Gani \\ Syahida Zubir F, Saniasiaya J, Abdul Gani H. Aural polyp with facial asymmetry in an unfortunate infant. Malays Fam Physician. 2021;16(1);133-135. \\ https://doi.org/10.51866/cr1070
}

\section{Keywords:}

rhabdomyosarcoma, temporal bone, facial palsy

\section{Authors:}

\section{Jeyasakthy Saniasiaya}

(Corresponding author)

Department of Otorhinolaryngology

Faculty of Medicine, University of

Malaya, Kuala Lumpur, Malaysia

Email: shakthy_18@yahoo.com

\section{Farah Syahida Zubir}

Department of Otorhinolaryngology

Hospital Tuanku Jaáfar, Seremban

Negeri Sembilan, Malaysia

Department of Otorhinolaryngology Faculty of Medicine, University of Malaya, Kuala Lumpur, Malaysia

\section{Haslinda Abdul Gani}

Department of Otorhinolaryngology Hospital Tuanku Jaáfar, Seremban

Negeri Sembilan, Malaysia

\begin{abstract}
Temporal bone rhabdomyosarcoma is an aggressive entity that simulates chronic otitis ear infection. It is the most common soft tissue sarcoma amongst pediatric patients. Herein, we would like to report a case of temporal bone rhabdomyosarcoma involving a 2-year-old boy who presented with a one-month history of otorrhea with facial asymmetry. Early treatment led to remission of this severe neoplasm.
\end{abstract}

\section{Introduction}

Rhabdomyosarcoma (RMS) is the third most common neoplasm in childhood, behind only neuroblastoma and nephroblastoma. ${ }^{1}$ Approximately $35 \%$ of all pediatric RMS cases occur within the head and neck, with orbit being the most common site. Involvement of the ear and the temporal bone region, albeit rare, have been reported amongst children. ${ }^{2}$ Clinical presentation of temporal bone RMS may resemble features of chronic suppurative otitis media, ${ }^{2}$ which may contribute to its delayed diagnosis. ${ }^{3}$ Advanced disease, however, will present with cranial nerve palsies and intracranial extension features. ${ }^{4}$

\section{Case Report}

A previously healthy 2-year-old-boy was referred to our center with a 1-month history of left otorrhea and facial asymmetry. According to his mother, the ear discharge was persistent despite being treated multiple times with ear drops and antibiotics. There were no signs of otalgia and the child remained afebrile. The mother, however, noticed left facial asymmetry two weeks later with no other signs of meningism or raised intracranial pressure. Besides that, the child was active and tolerating orally well.

Upon assessment, the child was comfortable under room air. Otoscopic examination revealed a polypoidal, fragile mass occupying the entire ear canal with purulent discharge. Facial asymmetry was lower motor neuron HouseBrackman Grade III. Besides that, other nose and throat examinations were unremarkable. The child was admitted and was commenced on intravenous Ceftriaxone and intravenous steroids. High-resolution computed tomography (CT) temporal bone revealed soft tissue density filling the entire left external and middle ear cavities with extensive erosion of ossicles, scutum, tegmen tympani, facial canal (both tympanic and mastoid segment) and mastoid air cells (Fig.1). Mastoid exploration was carried out, which revealed an extensive mass occupying the mastoid cavity and middle ear, causing erosion of the mastoid cortex, suprameatal and posterior meatal wall. Histopathological examination of the mass showed fragments of polypoidal tissue covered by squamous epithelium infiltrated by atypical cells exhibiting large hyperchromatic and pleomorphic cells with prominent nucleoli (Fig.2). These cells also express Desmin and Myogenin, negative for LCA, PanCK and S100 with proliferative index as high as $90 \%$, which is consistent with embryonal rhabdomyosarcoma. The parents were counselled and the child was referred to the oncology unit for chemoradiotherapy. The child underwent 23 fractions of radiotherapy under general anesthesia. Upon subsequent imaging and follow-up, the disease is stable with no evidence of recurrence.

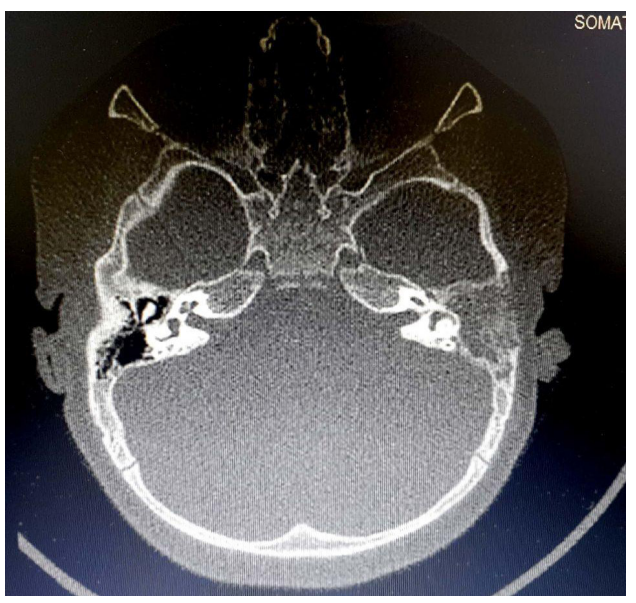

Figure 1. 


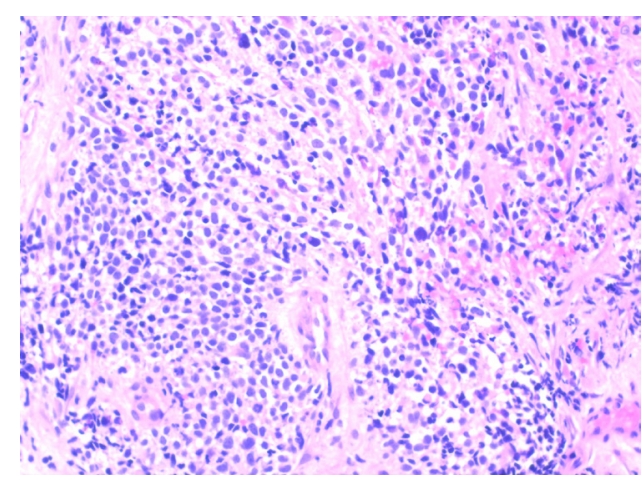

Figure 2.

\section{Discussion}

RMS is the most common soft-tissue malignancy seen in childhood. ${ }^{5}$ It may originate in any anatomical site, occurring predominantly in the head and neck regions, orbits, skull base, nasal cavity, and nasopharynx, where there is little or no musculoskeletal tissue. ${ }^{6}$ It has a bimodal age distribution, with the first peak between ages 3 and 5 years and the second peak between ages 14 and 18 years.

A retrospective study of children with temporal bone RMS found that the mean time between symptom onset and diagnosis was 7 weeks and the most common manifestation $(66 \%$ of patients) was otitis media unresponsive to treatment. ${ }^{8}$ According to Prat and Gray, who analyzed symptoms of RMS of the middle ear in 50 patients, $54 \%$ presented with polypoid masses in the external auditory canal, $40 \%$ with otorrhea, $30 \%$ with otorrhagia, $22 \%$ with otalgia, and $14 \%$ with facial nerve palsy. ${ }^{9}$ In our patient, he presented with unresolving otorrhea which may mimic chronic otitis media. However, it was when he developed facial asymmetry that a more serious explanation was suggested. Our initial differential diagnosis for this patient includes cholesteatoma, RMS and Langerhans cells histiocytosis (LCH).

Cholesteatoma in the pediatric population demonstrated an annual incidence of 2.9 per 100000 children. ${ }^{1}$ In parallel with increasing awareness of cholesteatoma, with the aid of otoscopic and endoscopic ear examination, children with congenital cholesteatoma are usually asymptomatic. Unlike RMS, cholesteatoma presents with a white mass behind an intact ear drum, or a deep retraction pocket with or without granulation and skin debris $^{2}$ Although facial nerve palsy can occur as a complication of cholesteatoma, it has been reported only in approximately 4.3\% of patients. ${ }^{3}$ In children with temporal bone RMS, though, the incidence is higher. Durve et al reported a case series in which 9 out of 14 (64\%) children with RMS of ear and temporal bone had facial nerve paralysis as part of their presentation. $^{4}$

It is a common practice in the treatment of children to perform imaging prior to biopsy so as to avoid unwarranted complications such as a torrential bleed from a vascular tumor. Temporal bone CT with contrast is the imaging of choice as it better delineates bony involvement. Magnetic resonance imaging (MRI) with contrast is most specific for diagnosing soft-tissue tumors and assessing meningeal invasion. ${ }^{8}$ The imaging characteristics for RMS and $\mathrm{LCH}$ are similar, but the primary site of involvement is different. ${ }^{10}$ According to Chevalier et al, the most common subsite of the temporal bone involved for LCH is mastoid while for RMS it is typically confined to the anterior part of the temporal bone. RMS demonstrates a homogeneous lesion with mild contrast enhancement in CT whilst on MRI it typically appears as a homogenous mass, isointense or minimally hyperintense relative to muscle on T1-weighted images and hyperintense relative to both muscle and fat on T2-weighted images, with postcontrast images showing enhancement of the tumor. ${ }^{11}$ As RMS does not arise from the bone, bony erosion is considered a poor prognostic sign.

RMS of the head and neck region falls into three categories: $51 \%$ of cases occur in the parameningeal region (nose, paranasal sinuses, middle ear and mastoid); $24.5 \%$ of cases present in the non-parameningeal region (parotid gland, oral cavity and larynx); and $24.5 \%$ of cases are in the orbit. RMS of the ear is rare, with only approximately $3 \%$ of patients presenting with the middle ear disease. In this case, RMS occupies the middle ear and mastoid. Histologically, RMS resembles normal skeletal muscle before innervation, and there are several pathologic subtypes of RMS: embryonal, botryoid, alveolar, pleomorphic, spindle cell, and anaplastic variants. ${ }^{12}$ The embryonal RMS comprises approximately $60-70 \%$ of RMS cases, ${ }^{13}$ which incidentally is our patient's histology. Embryonal subtypes have a better prognosis compared to other subtypes.

Complete surgical resection of the lesion may not only be technically difficult, but may result in significant cosmetic deformity ${ }^{14}$ Recent trends suggest that radical resection procedures are deemed redundant as the advent 
and advancement of chemoradiotherapy has dramatically improved the survival rate. ${ }^{2}$ Indeed, survival rates have improved so much that the International Society of Pediatric Oncology have been able to complete remission in $91 \%$ of patients with non-metastatic disease. ${ }^{15}$

\section{Conclusion}

Rhabdomyosarcoma of the temporal bone is an aggressive tumor which may mimic chronic otitis media. High index of suspicion is warranted in the context of persistent otitis media unresponsive to medical treatment. Immediate imaging followed by mastoid exploration is therefore recommended with presence of mass in external auditory canal with facial asymmetry. Early diagnosis and prompt management with multimodal therapy offers the best outcome amongst pediatric temporal bone rhabdomyosarcoma.

\section{How does this paper make a difference to general practice?}

- Presentation of otorrhea with facial asymmetry should alert physician of a more serious condition such as malignancy

- Persistent otorrhea with aural polyp in children should be investigated thoroughly, which should include biopsy and imaging

- In addition to histopathological investigations, immunohistochemical study is salient for accurate diagnosis

- Prompt investigation, diagnosis and management has revealed good prognosis

\section{References}

1. Stiller CA, Parkint DM. International variations in the incidence of childhood softtissue sarcomas. Paediatr Perinat Epidemiol. 1994;8(1):107-119.

2. Durve DV, Kanegaonkar RG, Albert D, Levitt G. Paediatric rhabdomyosarcoma of the ear and temporal bone. Clin Otolaryngol Allied Sci. 2004;29(1):32-37.

3. Alho O-P, Teppo H, Mäntyselkä P, Kantola $S$. Head and neck cancer in primary care: presenting symptoms and the effect of delayed diagnosis of cancer cases. Cmaj. 2006;174(6):779-784.

4. Ragab A, Abdulber Fakoury M, Kassouma J, Moustafa K, Al Salem FM. Botryoid rhabdomyosarcoma in mastoid and middle ear in a 4-year-old boy: A rare case report. Hamdan Med J. 2018;11(3):130.

5. Jan MMS. Facial paralysis: a presenting feature of rhabdomyosarcoma. Int J Pediatr Otorhinolaryngol. 1998;46(3):221-224.
6. Arita K, Sugiyama K, Tominaga A, Yamasaki F. Intrasellar rhabdomyosarcoma: case report. Neurosurgery. 2001;48(3):677-680.

7. Vlaški L, Vučković N, Dragičević D, Kljajić $\mathrm{V}$, Seničar S. Prolonged peripheral facial nerve paralysis in a child - think of temporal bone rhabdomyosarcoma: case report. Open Med. 2014;9(2):226-230.

8. Sbeity S, Abella A, Arcand P, Quintal MC, Saliba I. Temporal bone rhabdomyosarcoma in children. Int J Pediatr Otorhinolaryngol. 2007;71(5):807-814.

9. Prat J, Gray GF. Massive neuraxial spread of aural rhabdomyosarcoma. Arch Otolaryngol. 1977;103(5):301-303.

10. Chevallier KM, Wiggins RH, Quinn NA, Gurgel RK. Differentiating pediatric rhabdomyosarcoma and langerhans cell histiocytosis of the temporal bone by imaging appearance. Am J Neuroradiol. 2016;37(6):1185-1189.
11. Lee JH, Lee MS, Lee BH, et al. Rhabdomyosarcoma of the head and neck in adults: MR and CT findings. Am J Neuroradiol. 1996;17(10):1923-1928.

12. Vegari S, Hemati A, Baybordi $H$, Davarimajd L, Chatrbahr G. Embryonal Rhabdomyosarcoma in Mastoid and Middle Ear in a 3-Year-Old Girl: A Rare Case Report. Case Rep Otolaryngol. 2012;2012(Figure 2):1-3.

13. Vegari S, Hemati A, Baybordi $\mathrm{H}$, Davarimajd L, Chatrbahr G. Embryonal rhabdomyosarcoma in mastoid and middle ear in a 3-year-old girl: a rare case report. Case Rep Otolaryngol. 2012;2012.

14. Wiatrak BJ, Pensak ML. Rhabdomyosarcoma of the ear and temporal bone. Laryngoscope. 1989;99(11):1188-1192.

15. Maurer HM, Gehan EA, Beltangady M, et al. The intergroup rhabdomyosarcoma study-II. Cancer. 1993;71(5):1904-1922. 